\title{
Liver Tissue Segmentation In Multiphase CT Scans Using Cascaded Convolutional Neural Networks
}

\author{
Farid Ouhmich • Vincent Agnus • Vincent Noblet • \\ Fabrice Heitz • Patrick Pessaux
}

Received: date / Accepted: date

\begin{abstract}
Purpose: We address the automatic segmentation of healthy and cancerous liver tissues (parenchyma, active and necrotic parts of hepatocellular carcinoma (HCC) tumor) on multiphase CT images using a deep learning approach. Methods: We devise a cascaded convolutional neural network based on the U-Net architecture. Two strategies for dealing with multiphase information are compared: single-phase images are concatenated in a multi-dimensional features map on the input layer, or output maps are computed independently for each phase before being merged to produce the final segmentation. Each network of the cascade is specialized in the segmentation of a specific tissue. The performances of these networks taken separately and of the cascaded architecture are assessed on both single-phase and on multiphase images. Results: In terms of dice coefficients, the proposed method is on par with a state-of-the-art method designed for automatic MR image segmentation and outperforms previously used technique for interactive CT image segmentation. We validate the hypothesis that several cascaded specialized networks have a higher prediction accuracy than a single network addressing all tasks simultaneously. Although, the portal venous
\end{abstract}

Farid Ouhmich

Nouvel Hôpital Civil, Institut Hospitalo-Universitaire de Strasbourg, 1 place de lHôpital, 67000 Strasbourg, France Tel.: +33 390413646

E-mail: farid.ouhmich@ihu-strasbourg.eu

Vincent Agnus

Nouvel Hôpital Civil, Institut Hospitalo-Universitaire de Strasbourg, 1 place de lHôpital, 67000 Strasbourg, France Tel.: +33 390413646

Orcid : https://orcid.org/0000-0001-9554-6492

E-mail: vincent.agnus@ihu-strasbourg.eu

Vincent Noblet

ICube UMR 7357, University of Strasbourg, CNRS, FMTS, 300 bd Sébastien Brant, 67412 Illkirch, France

E-mail: vincent.noblet@unistra.fr

Fabrice Heitz

ICube UMR 7357, University of Strasbourg, CNRS, FMTS, 300 bd Sébastien Brant, 67412 Illkirch, France

E-mail: fabrice.heitz@unistra.fr

Patrick Pessaux

Department of Hepato-Biliary and Pancreatic Surgery, Nouvel Hôpital Civil, Institut Hospitalo-Universitaire de Strasbourg, 1 place de lHôpital, 67000 Strasbourg, France

E-mail: patrick.pessaux@chru-strasbourg.fr 
phase alone seems to provide sufficient contrast for discriminating tumors from healthy parenchyma, the multiphase information brings significant improvement for the segmentation of cancerous tissues (active vs necrotic part). Conclusion : The proposed cascaded multiphase architecture showed promising performances for the automatic segmentation of liver tissues, allowing to reliably estimate the necrosis rate, a valuable imaging biomarker of the clinical outcome.

Keywords Hepatocellular Carcinoma · Multiphase CT · Semantic Segmentation · Fully Convolutional Networks (FCNs) · Liver Tissues

\section{Introduction}

Liver cancer is the sixth most common cancer worldwide, and the third most common cause of cancer death according to the Global Cancer Observatory [6]. Among the different types of primary liver cancers, the hepatocellular carcinoma is the most frequent and the most lethal one. Contrast Enhanced Computed Tomography (CECT) is one of the most informative image modality for monitoring liver cancer $[14,25]$. The main advantage brought by the CECT compared to Non Enhanced CT (NECT) is its ability to investigate the dynamics of tissue vascularization and the vessel structure [23]. However, as a prerequisite to a quantitative analysis of such temporal sequences, an efficient deformable registration method is needed to compensate for respiratory motion between the different phases $[14,22]$.

In the clinical routine, the detection of lesions as well as the estimation of their size and number are still broadly performed using visual inspection of CT and MR images, which can be subjective, operator dependent and prone to diagnosis misinterpretations because of the high inter-tumor variability. The high intra-tumoral heterogeneity should also be considered when characterizing the cancer phenotype [19]. In radiomics studies, this can be achieved by differentiating active from necrotic tissue within the tumor $[25,10]$ using shape and texture information. This differentiation can also be useful to compute the necrosis rate, which is a valuable imaging biomarker for patients follow-up, since it has been shown to be correlated with survival after treatment [20].

All these observations underline the need for automatic and reliable tools dedicated to tumor segmentation and active and necrotic part discrimination in order to finely characterize liver cancer.

\subsection{Related Work}

The segmentation of the liver and of its lesions remains a challenging task, mainly due to the high anatomical shape variability and to the fact that liver may be in contact with neighboring structures of similar intensities. In addition to that, diseases affecting the liver may have a large impact on its textural properties [17].

Intensity based techniques were proposed either by learning the intensity distribution from training samples in order to pre-segment the region of interest [7], or simply by using thresholding techniques [13]. Machine learning methods were also proposed in combination with the aforementioned techniques to segment the liver [26] and its lesions [8]. Statistical shape models were considered in order to capture the shape of the liver, while coupling them with deformable model [5] or graph-cuts [24]. Methods based on probabilistic atlases are an alternative to incorporate $a$ priori knowledge about location and shape of the liver, as well as information about surrounding anatomical structures [15]. 
More recently, deep learning approaches have led to major breakthroughs in medical image analysis. They have been recognized for their ability to encode complex morphological and texture information in images by directly using raw pixel intensities as inputs, whereas traditional machine learning methods required carefully hand-crafted features to achieve descent results $[16,28]$. Deep learning networks achieved state-of-the-art results in many medical-related applications, including detection, classification, segmentation, localization and registration $[11,16]$. These performances have been reached, even with a small number of training cases, thanks to smart architecture choices, data augmentation, or transfer learning [31]. Fully convolutional networks (FCNs) are now widely used for medical applications [9], in particular for liver and lesions segmentation $[2,3,27,30]$. They significantly outperform the standard methods mentioned above.

Segmentation using FCNs was popularized by the U-Net network initially introduced to segment cells on histopathological slices [21] and afterwards applied to liver and lesion segmentation [2]. More recently, a cascaded version of the U-Net network was used for both liver and lesion segmentation [3]. Only a few studies exploited the multiphase information using FCNs [27] and to our knowledge only one contribution tackled the delineation of both active and necrotic parts within the lesions, but using MR images [30].

\subsection{Contributions}

In this work, we applied deep learning-based semantic segmentation to delineate the liver and its lesions in contrast-enhanced $\mathrm{CT}$ sequences, while discriminating the necrotic from the active parts. This task was previously addressed in the context of MR images [30]. We evaluate the impact of using either single-phase or multiphase CT images on the performance of different FCNs architectures. We showed that portal-phase images alone provide enough contrast for discriminating lesions from healthy parenchyma, while multiphase information brings significant improvement for the segmentation of active and necrotic tumor parts. Finally, our results support the hypothesis that combining several specialized networks in cascade leads to better performance than a single versatile network addressing all tasks simultaneously [3].

\section{Material and Methods}

\subsection{Database}

Due to the lack of publicly available datasets providing segmentation of active and necrotic tissues within hepatic lesions, we used an in-house database $(D b)$ containing ground truth $2 \mathrm{D}$ annotations for three classes, namely the healthy parenchyma, the active and the necrotic parts of the lesions. A subset of $D b$ was already used in a previous work on the semi-automatic segmentation of liver tissue [4].

The database is composed of data from seven patients suffering from hepatocellular carcinoma (HCC) that underwent from one to three contrast enhanced computed tomography (CECT) examinations (in plane resolution ranging from 0.66 to $0.97 \mathrm{~mm}$, slice thickness ranging from 0.7 to $1.25 \mathrm{~mm}$ ), resulting in a total number of $13 \mathrm{CT}$ sequences. Each examination is composed of one image before contrast agent injection (NECT), and two contrast enhanced images reflecting the arterial $(\mathrm{AR})$ phase $(\approx 25-30$ seconds after injection $)$ and the portal venous $(\mathrm{PV})$ phase $(\approx 60-70$ seconds after injection). For each CT sequence, four experts have manually segmented the three 
classes on eight slices regularly sampled across the tumor, resulting in a total number of 104 labeled slices. Expert annotations were fused using the STAPLE algorithm to reach a consensus [29]. The manual segmentation task was performed on the PV images, which exhibit the best contrast between the different tissues.

\subsection{Data pre-processing}

As a prerequisite for the use of multiphase information [14], respiratory motion between the different phase images was corrected using a diffeomorphic deformable registration algorithm [1]. The PV image was considered as the reference image in the registration process, following [4].

Images from $D b$ are of heterogeneous sizes and voxel resolutions. Since FCN require a standardized input size, a scaling transformation was applied to all images to enforce isotropic resolution $\left(0.97 \mathrm{~mm}^{2}\right)$ and a fixed $512 \times 512$ size.

An intensity normalization procedure similar to [3] was then applied. We only retained Hounsfield Unit (HU) intensities within the range $[-100 \mathrm{HU}, 400 \mathrm{HU}]$, corresponding to typical radiodensity values of the liver, to reduce the influence of neighbouring organs. Contrary to [3], we did not implement slice-wise histogram equalization since it may be biased by the different proportion of liver present in each slice. Instead, we considered a linear mapping of the retained HU values to the $[0,1]$ interval.

\subsection{Cascaded Networks}

As in [3], we decompose the segmentation task in a hierarchical manner by cascading several networks relying on the U-Net architecture, each of them being specialized in the segmentation of a specific tissue. In the sequel, a given single phase elementary network will be referred to by the input phase and the target class. For example PV-Lesion (see figure 1) corresponds to a network dedicated to segment lesions using PV images as input, while AR-Necrosis refers to the network dedicated to the segmentation of the active $v s$ necrotic parts of the lesions using AR images as an input.

Two strategies were investigated to exploit multiphase information. The first one, referred as DMP (Dimensional MultiPhase) strategy, consists in concatenating single-phase images in a multidimensional feature map used as the input to the network (see figure 2). The second one, referred as MPF (MultiPhase Fusion) strategy, consists in processing each phase independently and then merges the output maps (by simple addition) to obtain the final segmentation (see figure 3). Only the AR and PV phases were considered in the DMP and MPF networks because NECT does not provide enough inter-tissue contrast.

The cascaded architecture is depicted in figure 4 and can be composed of either single or multiphase U-net sub-networks. Unlike the original cascaded architecture [3], which cropped the original image to the predicted liver area after the first step, we decided to keep the same image size all throughout the networks. This choice was motivated by the fact that cropping the image would require to resample it in order to fit the fixed input size of the network, which would alter its isotropic resolution.

To validate the hypothesis suggesting that cascaded specialized networks perform better than a versatile network addressing all tasks simultaneously [3], we consider the original U-net architecture (denoted $\{\cdot\}$-Full) to simultaneously predict the three classes of liver tissues (see figure 5 ). This network is evaluated for both single phase and multiphase conditions. To ensure that there was no 


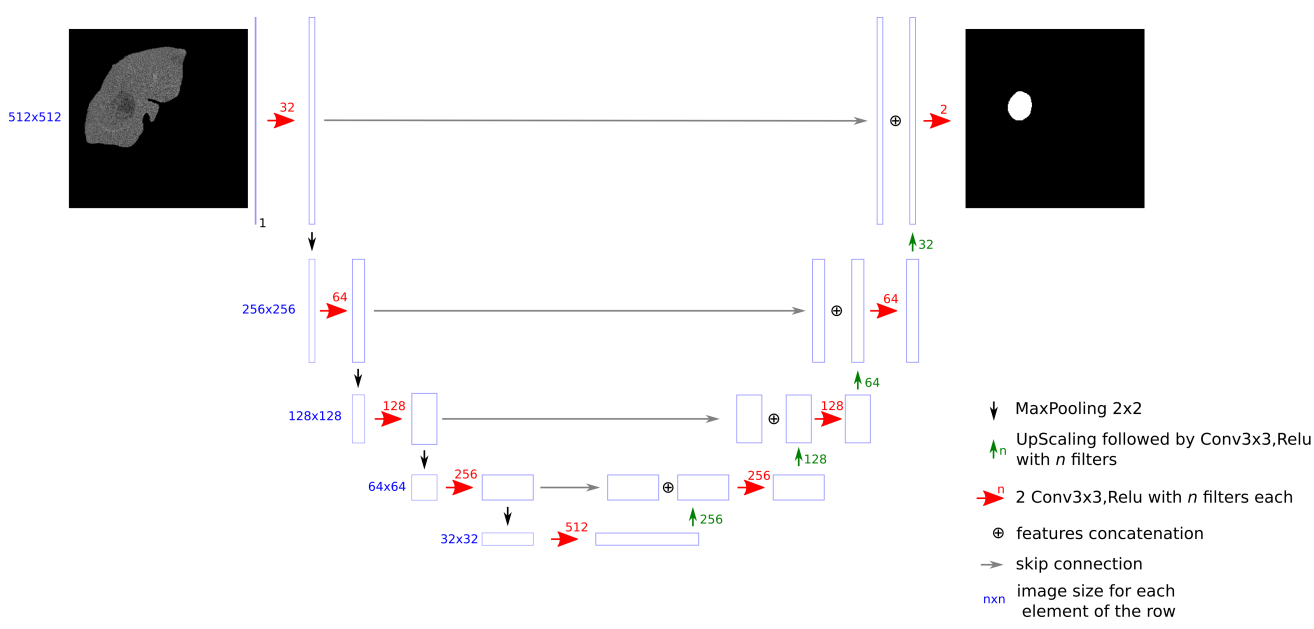

Fig. 1 PV-Lesion network used to segment lesions within the liver with a PV image as input.

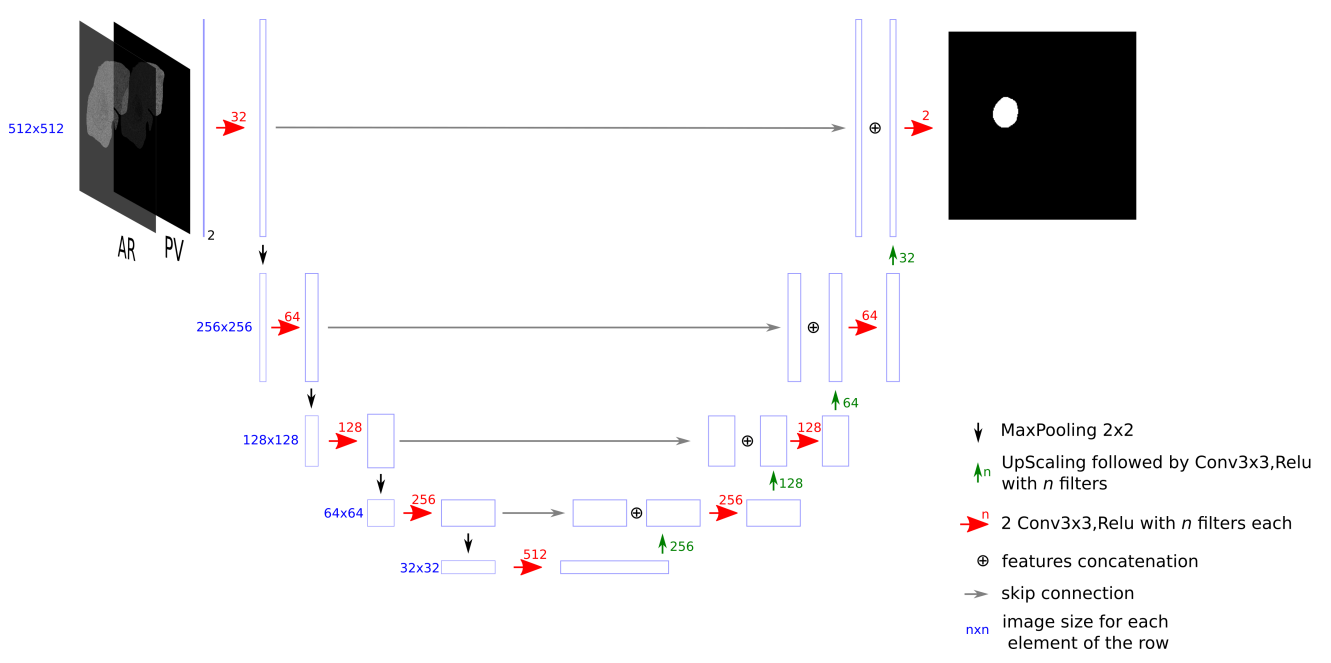

Fig. 2 DMP-Lesion network that combines the AR and the PV images as an input to segment the lesions within the liver. Here, the two channels are considered as features for the first layer.

bias in favor of the cascaded networks in the comparison, the cascaded networks were imposed to have a smaller number of parameters than the versatile one (which had a total of $32 \mathrm{M}$ parameters). $8 \mathrm{M}$ parameters were specified for all cascaded networks, apart from MPF- \{\} , with 16M parameters (fusion of two networks with $8 \mathrm{M}$ parameters each).

\subsection{Training}

FCNs have proven their ability to operate well even when trained on small databases. A data augmentation step is generally required to avoid overfitting and to generalize better on unseen cases. 


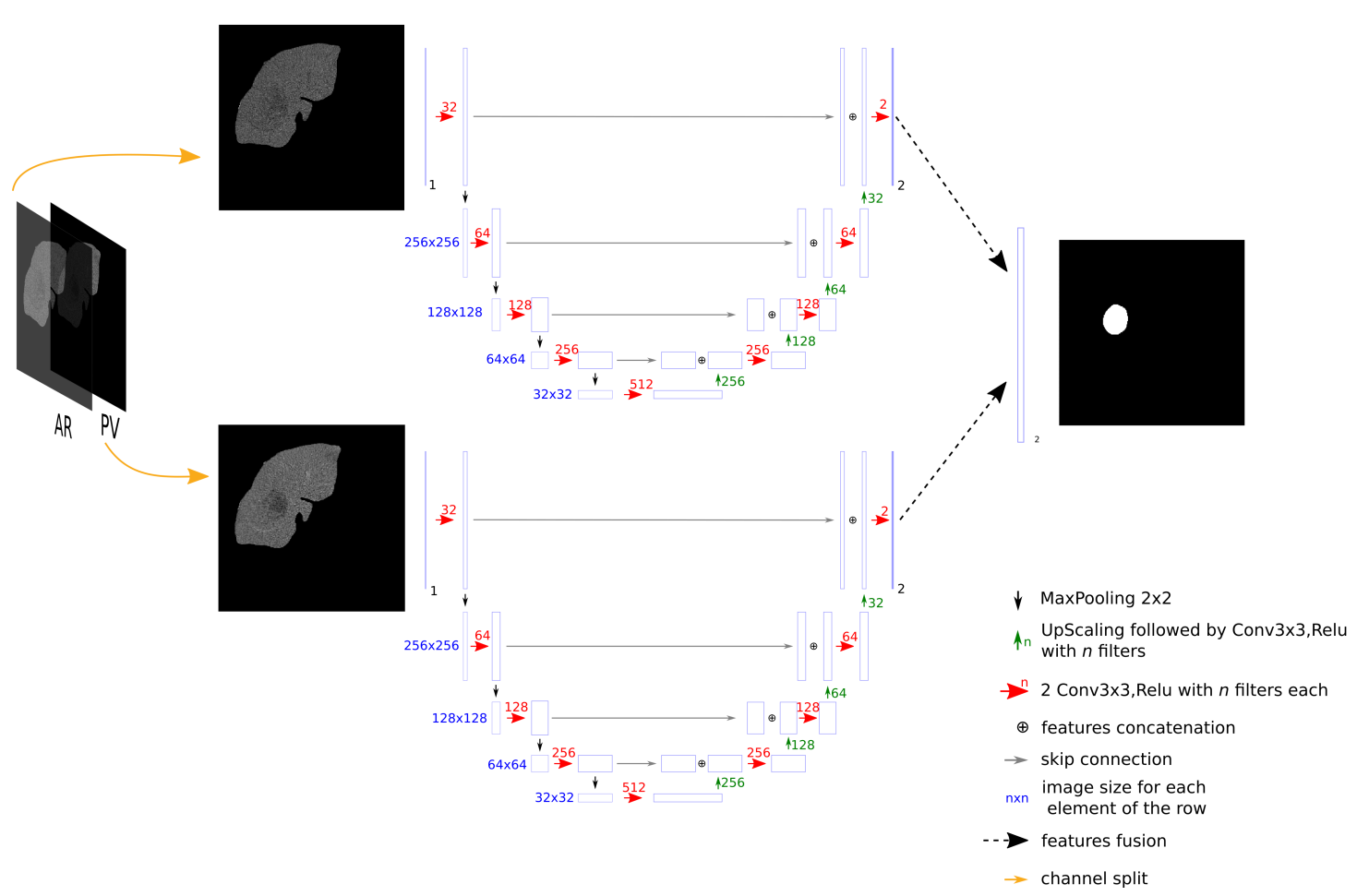

Fig. 3 MPF-Lesion network: initially, AR and PV images are processed separately. The resulting maps are merged (by simple addition) at the end.

In our case, for each input image, ten new images were randomly generated using the following settings:

- Rotation $\left(90^{\circ}, 180^{\circ}, 270^{\circ}\right)$.

- Horizontal/Vertical flip.

- Horizontal/Vertical shift in range $[0,0.1]$ of the image size.

- Addition of Gaussian noise $(\mu=0, \sigma=3 \mathrm{HU})$.

We chose to consider a moderate noise variance since the inter-class difference is generally smaller than $20 \mathrm{HU}$.

A leave-one-patient-out cross validation process was used during the training to ensure that no slices from the same patient were present in both training and test sets. The networks were implemented using Keras with a TensorFlow backend and trained on a single NVIDIA GTX 1070 with 8GB VRAM. We used the Adam optimizer [12] with a learning rate of $10^{-4}$ and a decay of $10^{-5}$. No additional dropout layers were used since it appeared to decrease the performance, probably due to the small number of images available. The softmax function is considered for the final layer of the different networks. To cope with the imbalanced classes problem, we considered the weighted cross-entropy as the cost function to optimize the weights of the networks, as suggested in [18]. The segmentation quality was evaluated with the Dice similarity coefficient (DSC), which is commonly used for the assessment of semantic segmentation $[3,30,2,4]$. 

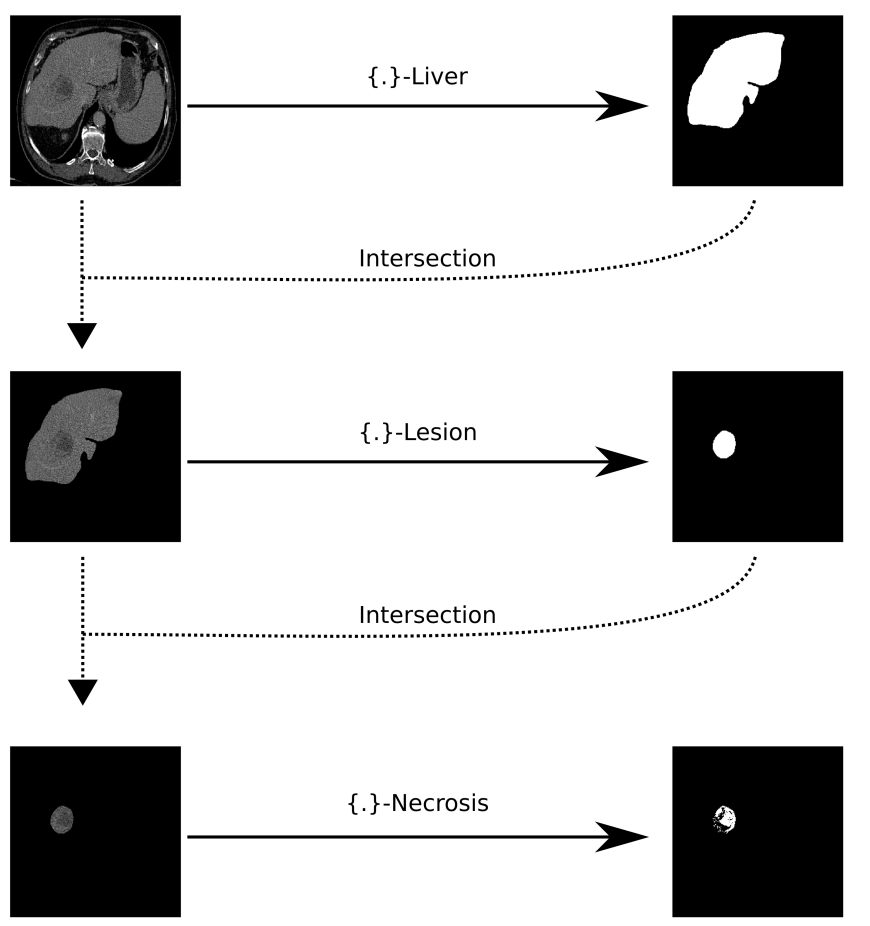

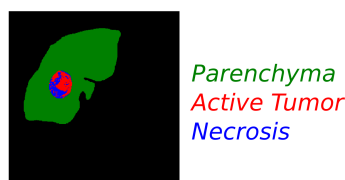

Overlap of predicted binary masks

Fig. 4 Cascaded Network: the first network takes as input a CT image and segments the liver. The resulting segmentation map is used to remove non-liver pixels in the input data of the second network which performs the segmentation of lesions. The last network segments the necrosis within the lesions. The three binary masks are combined in the final segmentation map.

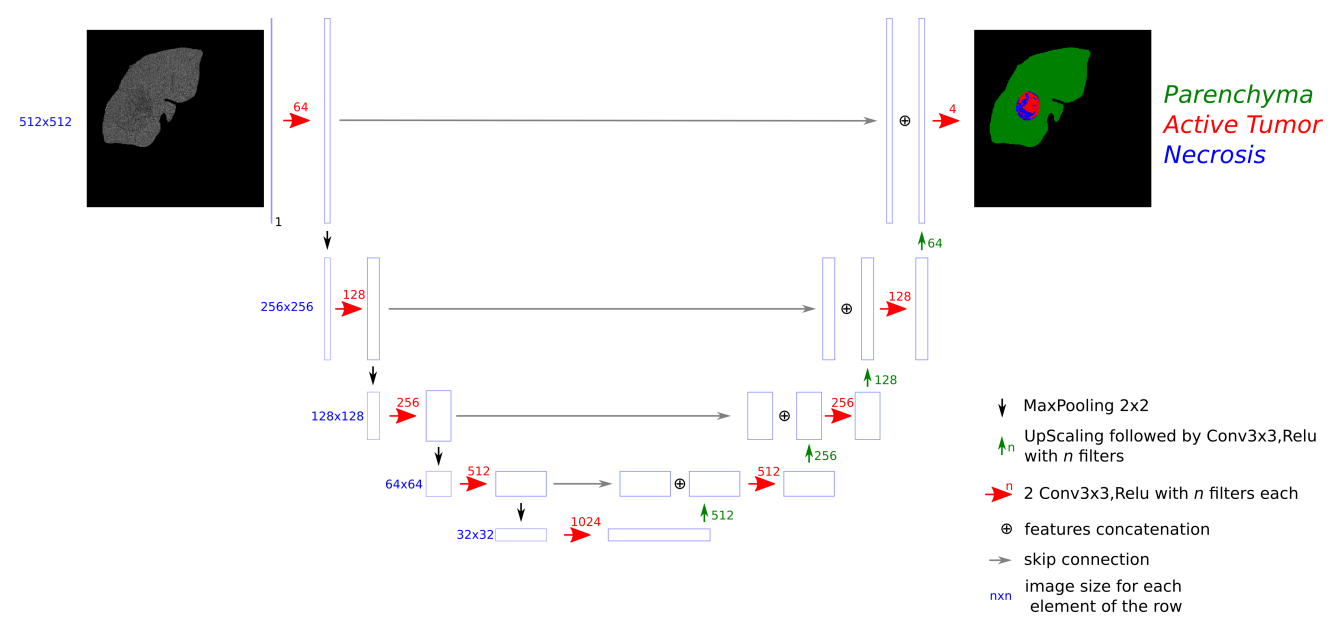

Fig. 5 AR-Full refers to the network trained with AR images as input (values outside the liver are masked), and that outputs a label map, with parenchyma, active and necrotic parts annotated. 


\section{Experiments and Results}

Mean DSCs were computed over all slices for each target class. The different methods were statistically compared using the Wilcoxon signed paired rank tests, since slice-wise DSCs did not follow a normal distribution. We first compared $\{\mathrm{NECT}, \mathrm{AR}, \mathrm{PV}, \mathrm{DMP}, \mathrm{MPF}\}$-Liver networks to evaluate which phase allows better liver segmentation. We then trained (NECT, AR, PV, DMP, MCF\}-Lesion and $\{\mathrm{NECT}, \mathrm{AR}, \mathrm{PV}, \mathrm{DMP}, \mathrm{MCF}\}$-Necrosis networks separately on $D b$ by masking all values outside the liver using ground truth annotations in order to assess whether multiphase information is really useful for the segmentation. The results are given in table 1.

Multiphase performed significantly better than single-phase for segmenting the liver (DMP vs PV, $P=0.001 ;$ DMP vs AR, $P=0.005$, DMP vs NECT, $P<0.001)$ and the active part of the lesions (DMP vs PV, $P<0.001 ; \mathrm{DMP}$ vs AR, $P=0.003$; DMP vs NECT, $P<0.001$ ). When comparing single-phase alone, PV achieved significantly better DSCs than AR or NECT for all the segmentation tasks except for the liver segmentation. When comparing multiphase methods, DMP carries out significantly better than MPF for the segmentation of the liver (DMP vs MPF, $P=0.004$ ), the parenchyma (DMP vs MPF, $P<0.001)$ and the active part of the lesions (DMP vs MPF, $P=0.005)$.

Since both DMP-Lesion and DMP-Necrosis led to the best results, we combined them in a cascade as explained before, and compared it to both $\{$ NECT, AR, PV, DMP, MPF\}-Full networks and evaluate them in terms of liver tissue classification performance (experiments were conducted on images that were masked with the ground truth liver segmentation). The mean DSCs are reported in table 2. Examples of segmentation results are shown in figure 6.

Table 1 Segmentation results using single-phase vs multiphase methods on $D b$.

\begin{tabular}{|c|c|c|c|c|c|c|}
\hline \multicolumn{1}{|c|}{ Input } & Target & \multicolumn{5}{c|}{ Network } \\
\hline Raw CT & LECT & AR & PV & DMP & MPF \\
\hline True liver mask & Parenchyma & $81.1 \pm 27.7$ & $89.5 \pm 13.2$ & $88.7 \pm 11.4$ & $\mathbf{8 9 . 9} \pm \mathbf{1 5 . 6}$ & $88.2 \pm 16.0$ \\
\hline True liver mask & Lesion & $77.4 \pm 24.1$ & $77.4 \pm 20.2$ & $87.8 \pm 9.7$ & $\mathbf{8 8 . 5} \pm \mathbf{1 1 . 7}$ & $86.6 \pm 10.3$ \\
\hline True lesion mask & Necrosis & $67.5 \pm 15.5$ & $69.7 \pm 16.3$ & $77.8 \pm 12.4$ & $78.5 \pm 13.3$ & $\mathbf{7 8 . 8} \pm \mathbf{1 1 . 7}$ \\
\hline True lesion mask & Active Tumor & $65.6 \pm 20.4$ & $63.9 \pm 22.6$ & $71.6 \pm 20.7$ & $\mathbf{7 5 . 5} \pm \mathbf{1 7 . 4}$ & $73.2 \pm 18.6$ \\
\hline
\end{tabular}

Table 2 Segmentation results using $\{\cdot\}$-Full vs cascaded architectures on $D b$.

\begin{tabular}{|c|c|c|c|c|c|c|}
\hline Target & \multicolumn{5}{|c|}{ Network } \\
\hline & NECT-Full & AR-Full & PV-Full & DMP-Full & MPF-Full & Cascaded DMP \\
\hline Parenchyma & $85.3 \pm 14.9$ & $84.1 \pm 17.9$ & $87.0 \pm 19.0$ & $82.9 \pm 19.7$ & $87.9 \pm 15.9$ & $\mathbf{9 0 . 5} \pm \mathbf{1 3 . 2}$ \\
\hline Necrosis & $62.6 \pm 18.6$ & $63.5 \pm 21.5$ & $75.7 \pm 14.4$ & $73.7 \pm 14.1$ & $75.6 \pm 13.4$ & $\mathbf{7 5 . 8} \pm \mathbf{1 5 . 1}$ \\
\hline Active Tumor & $42.2 \pm 24.0$ & $43.2 \pm 26.1$ & $53.5 \pm 24.2$ & $51.3 \pm 25.6$ & $52.0 \pm 23.3$ & $\mathbf{5 9 . 6} \pm \mathbf{2 2 . 5}$ \\
\hline
\end{tabular}

The results highlight that the cascaded version performed significantly better than $\{\cdot\}$-Full networks for segmenting the active part of the lesion (Cascaded DMP vs PV-Full, $P=0.001$ ). From the resulting segmentation maps, we were also able to estimate the necrosis rate, which is commonly used for diagnosis and prognosis of the treatment outcome. Our workflow provided estimates of 
Table 3 Average patient-wise segmentation DSCs (on full agreement expert area) with the semi-interactive approach of [4] and the cascaded DMP method

\begin{tabular}{|c|c|c|}
\hline & semi-interactive method $[4]$ & ours \\
\hline Parenchyma & $\mathbf{9 3 . 7} \pm \mathbf{3 . 4}$ & $92.2 \pm 4.7$ \\
\hline Lesion & $90.7 \pm 6$ & $\mathbf{9 1 . 8} \pm \mathbf{4}$ \\
\hline Necrosis & $83.0 \pm 12.9$ & $\mathbf{8 3 . 6} \pm \mathbf{1 1 . 7}$ \\
\hline Active Tumor & $75.2 \pm 10.9$ & $\mathbf{8 2 . 0} \pm \mathbf{6 . 4}$ \\
\hline Necrosis rate error & $7.84 \pm 4.4$ & $\mathbf{7 . 1 0} \pm \mathbf{1 . 4}$ \\
\hline
\end{tabular}

this valuable biomarker with a mean error rate of $13.0 \%$, which is accurate enough for clinical application.

With our setup, we also achieved slightly better results than those obtained in [30] for the same task, on a different database of MR images. We evaluated our method on the same database used in [4], where a manual expert interaction was required for the segmentation phase, which is not the case in the present deep learning approach. To allow fair comparison, the evaluation was conducted on the areas where all the experts reached an agreement as in [4]. The mean patient-wise segmentation DSCs are depicted in table 3. Our method enabled a better segmentation of the lesions and both necrotic and active parts. Therefore, we were able to predict the patient-wise necrosis rate with a slightly better precision.

We finally used the complete cascaded network, as depicted in figure 4, which combines DMPLiver, DMP-Lesion and DMP-Necrosis to perform a fully automatic segmentation from the raw (unmasked) CT image. We reached average slice-wise DSCs of $78.3 \pm 22.1$ for the segmentation of the parenchyma, $50.6 \pm 24.6$ for the segmentation of the active part, and $68.1 \pm 23.2$ for the necrotic part of the lesions. As a by-product, we also provided a necrosis rate per patient with a mean error of $15.9 \%$. Examples of fully automatic segmentation results are given in figure 7 .

\section{Conclusion}

Following the intuition developed in [3], we validated the hypothesis that FCNs are able to learn the hierarchical structures present in a CT image, even when trained on small databases. It has been shown that several cascaded specialized FCNs yield more accurate results than a single network addressing all tasks simultaneously. For a fair comparison, this has been demonstrated on networks with a comparable number of parameters.

The segmentation accuracy obtained by our method is on par with the one reported in [30], which addresses the same task using a different database of MR images. The accuracy of the FCNbased automatic segmentation method outperforms the results reported in [4] on the same database, using an ensemble classifier and requiring manual expert interaction during the segmentation. As a by-product, we have also shown that the segmentation maps may be used to provide usable estimates of the necrosis rate, which is an important predictor of treatment outcome.

The use of multiphase information has been proven to be beneficial when segmenting active vs necrotic tumour parts. However, this requires an accurate registration of the different phases, which remains a challenging step. As an alternative, the single PV phase can be used, with reasonable results for the lesion segmentation. A previous study has already reported that multiphase information is helpful for lesion segmentation, but that good outcomes may be reached using AR images 

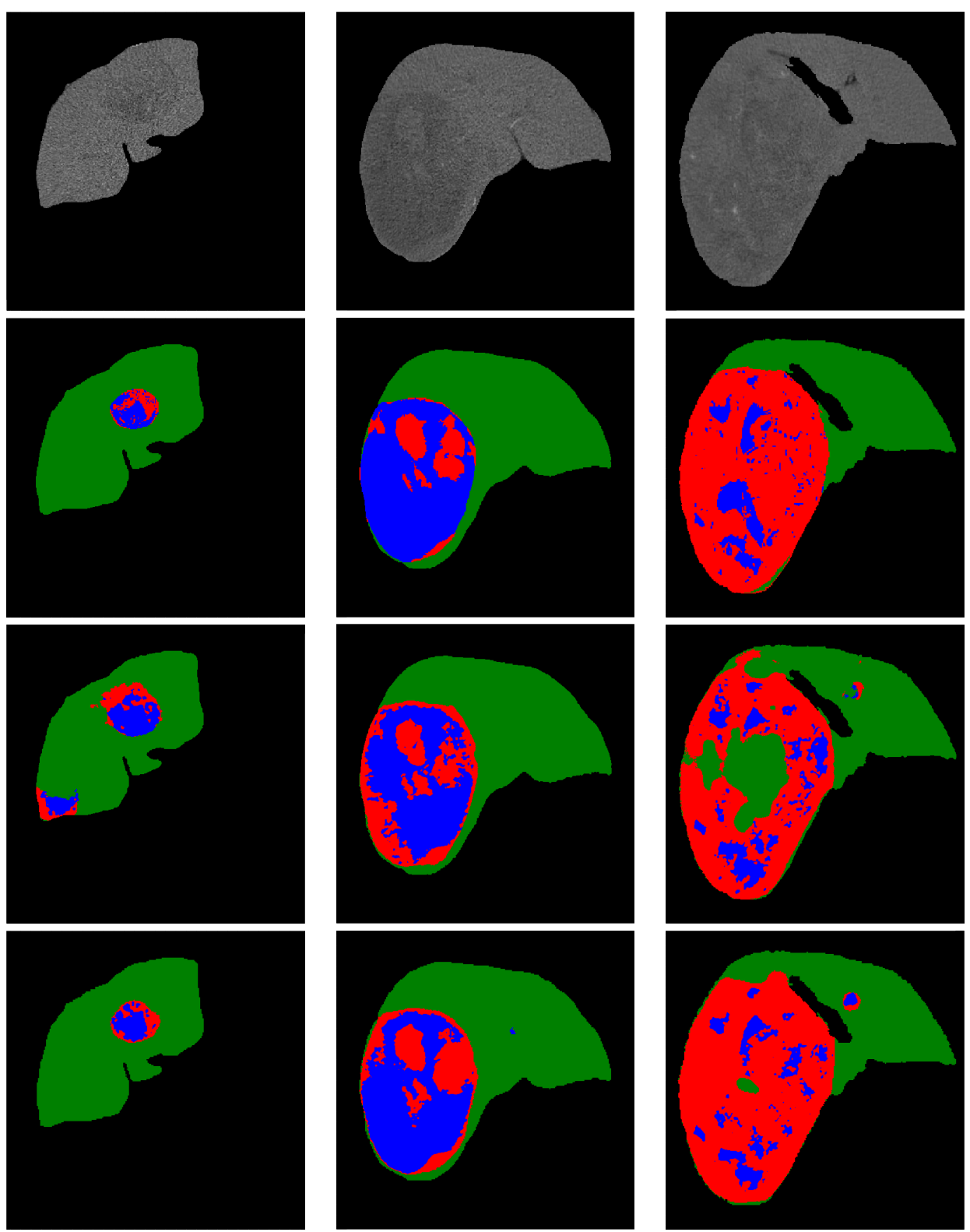

Fig. 6 From top to bottom : Raw images with HU values inside the liver, Ground truth, DMP-Full segmentation, Cascaded DMP segmentation

only [27]. This difference in the most appropriate phase for segmentation may be explained by the fact that our training database was only composed of HCC images whereas [27] was confronted with different tumor types, that respond differently to contrast agents. More interestingly, multiphase 

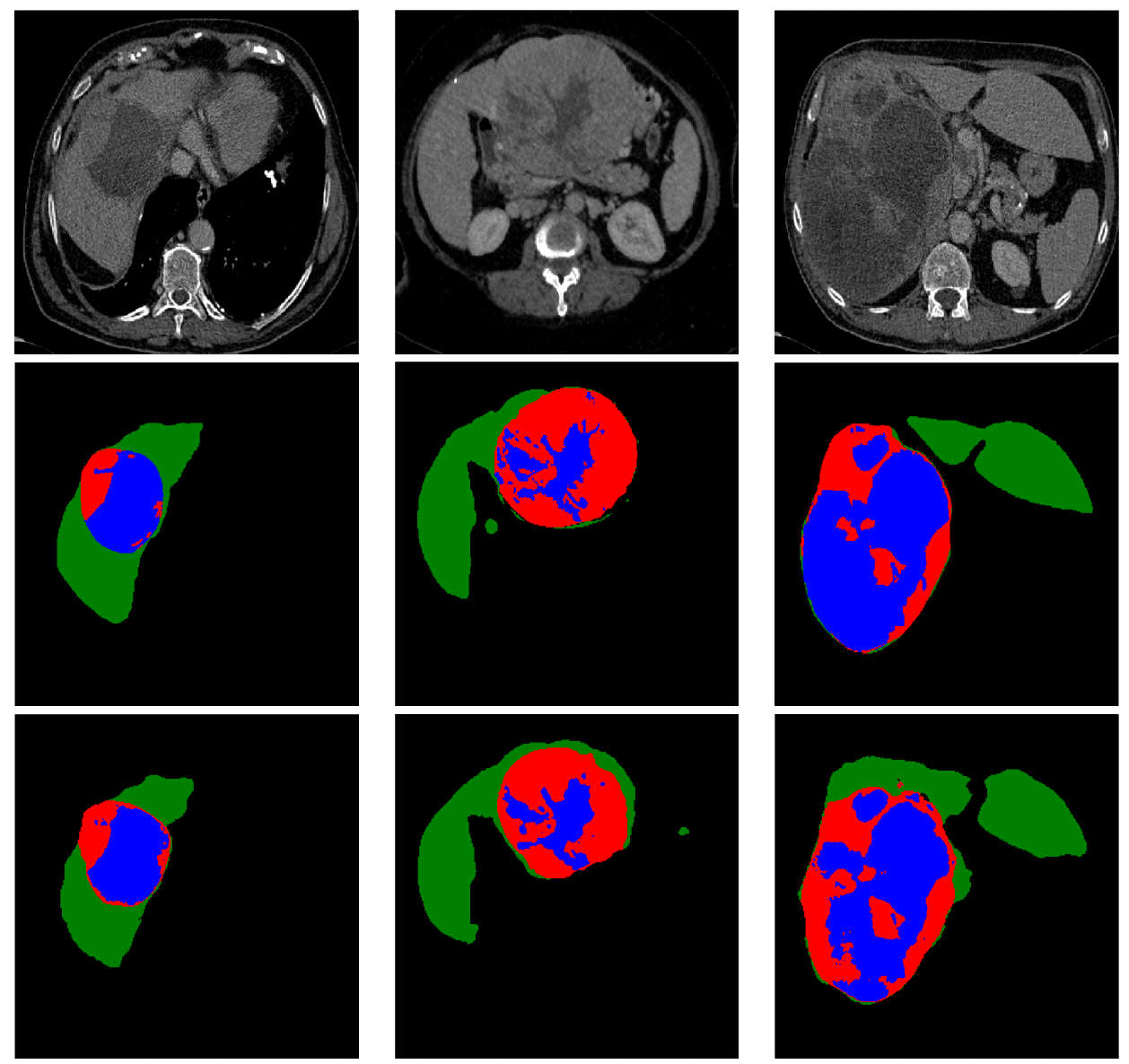

Fig. 7 From top to bottom : Raw images, ground truth and results of the fully automatic segmentation of liver tissue

information also significantly helped the segmentation of the liver. This may be explained by the high inter-class contrast present between lesion and parenchyma at PV-phase, leading PV-Liver to sometimes not consider lesions as part of the liver.

In this work, we have based our 3D segmentation on the segmentations of $2 \mathrm{D}$ slices. This choice was motivated by the will to limit the computational cost, but also by the difficulty to cope with the high variability of inter-voxels spacing in the $z$-direction. In addition, our training database only contained sparse annotations on 2D slices, which prevented a full 3D training. The use of full $3 \mathrm{D}$ information during the training, segmentation or in a post-processing phase is a perspective of this work. The benefit of using 3D-CRF in a post-processing step has for instance been shown for both liver and lesion segmentation in [3].

To our knowledge, we are the first to provide a method that fully automatically segment liver tissues on CT images. We conclude that automatic segmentation of liver tissue remains a challenging task, but our results prove that it can be tackled using fully convolutional networks. In addition, the 
resulting lesion segmentations can provide useful clinical information for patients follow-up, such as the necrosis rate.

\section{Compliance with Ethical Standards}

Funding : This study was funded by IHU Strasbourg through ANR grant 10-IAHU-0002.

Conflict of Interest: The authors declare that they have no conflict of interest.

Ethical standard : All procedures performed in studies involving human participants were in accordance with the ethical standards of the institutional and/or national research committee and with the 1964 Helsinki declaration and its later amendments or comparable ethical standards.

Informed consent: Informed consent was obtained from all individual participants included in the study.

\section{References}

1. Avants, B.B., Epstein, C.L., Grossman, M., Gee, J.C.: Symmetric diffeomorphic image registration with crosscorrelation: evaluating automated labeling of elderly and neurodegenerative brain. Med Image Anal 12(1), 26-41 (2008)

2. Ben-Cohen, A., Diamant, I., Klang, E., Amitai, M.: Deep Learning and Data Labeling for Medical Applications 10008, 77-85 (2016). DOI 10.1007/978-3-319-46976-8. URL http://link.springer.com/10.1007/ 978-3-319-46976-8

3. Christ, P.F., Ettlinger, F., Grün, F., Elshaera, M.E.A., Lipkova, J., Schlecht, S., Ahmaddy, F., Tatavarty, S., Bickel, M., Bilic, P., Rempfler, M., Hofmann, F., Anastasi, M.D., Ahmadi, S.A., Kaissis, G., Holch, J., Sommer, W., Braren, R., Heinemann, V., Menze, B.: Automatic Liver and Tumor Segmentation of CT and MRI Volumes using Cascaded Fully Convolutional Neural Networks pp. 1-20 (2017). URL http://arxiv.org/abs/1702.05970

4. Conze, P.H., Noblet, V., Rousseau, F., Heitz, F., de Blasi, V., Memeo, R., Pessaux, P.: Scale-adaptive supervoxelbased random forests for liver tumor segmentation in dynamic contrast-enhanced CT scans. International Journal of Computer Assisted Radiology and Surgery 12(2), 223-233 (2017). DOI 10.1007/s11548-016-1493-1. URL http://link. springer.com/10.1007/s11548-016-1493-1

5. Erdt, M., Steger, S., Kirschner, M., Wesarg, S.: Fast automatic liver segmentation combining learned shape priors with observed shape deviation. Proceedings - IEEE Symposium on Computer-Based Medical Systems pp. 249-254 (2010). DOI 10.1109/CBMS.2010.6042650

6. F. Bray, Jacques Ferlay, Isabelle Soerjomataram, Siegel;, R.L., Torre;, L.A., Jemal, A.: Global Cancer Statistics 2018: GLOBOCAN Estimates of Incidence and Mortality Worldwide for 36 Cancers in 185 Countries. CA: A Cancer Journal for Clinicians (2018). DOI 10.3322/caac.21492

7. Foruzan, A.H., Aghaeizadeh Zoroofi, R., Hori, M., Sato, Y.: Liver segmentation by intensity analysis and anatomical information in multi-slice CT images. International Journal of Computer Assisted Radiology and Surgery 4(3), 287-297 (2009). DOI 10.1007/s11548-009-0293-2

8. Freiman, M., Cooper, O., Lischinski, D., Joskowicz, L.: Liver tumors segmentation from CTA images using voxels classification and affinity constraint propagation. International Journal of Computer Assisted Radiology and Surgery 6(2), 247-255 (2011). DOI 10.1007/s11548-010-0497-5

9. Hu, Z., Tang, J., Wang, Z., Zhang, K., Zhang, L., Sun, Q.: Deep learning for image-based cancer detection and diagnosisA survey. Pattern Recognition 83, 134-149 (2018). DOI 10.1016/j.patcog.2018.05.014. URL https://doi.org/10.1016/j.patcog.2018.05.014

10. Jeong, W.K., Jamshidi, N., Felker, E.R., Raman, S.S., Lu, D.S.: Radiomics and radiogenomics of primary liver cancers. Clinical and Molecular Hepatology pp. 1-9 (2018). DOI 10.3350/cmh.2018.1007. URL http://www. e-cmh.org/journal/view.php?doi=10.3350/cmh.2018.1007

11. Ker, J., Wang, L., Rao, J., Lim, T.: Deep Learning Applications in Medical Image Analysis. IEEE Access 6, 9375-9379 (2017). DOI 10.1109/ACCESS.2017.2788044

12. Kingma, D.P., Ba, J.: Adam: A Method for Stochastic Optimization. AIP Conference Proceedings 1631, 58-62 (2014). DOI 10.1063/1.4902458. URL http://arxiv.org/abs/1412.6980

13. Kumar, S.S., Moni, R.S., Rajeesh, J.: Automatic liver and lesion segmentation: A primary step in diagnosis of liver diseases. Signal, Image and Video Processing 7(1), 163-172 (2013). DOI 10.1007/s11760-011-0223-y

14. Lee, J., Kim, K.W., Kim, S.Y., Shin, J., Park, K.J., Won, H.J., Shin, Y.M.: Automatic detection method of hepatocellular carcinomas using the non-rigid registration method of multi-phase liver CT images. Journal of X-Ray Science and Technology 23(3), 275-288 (2015). DOI 10.3233/XST-150487 
15. Li, C.Y., Wang, X., Eberl, S., Fulham, M., Yin, Y., Feng, D.: Fully automated liver segmentation for lowand high-contrast ct volumes based on probabilistic atlases. Proceedings - International Conference on Image Processing, ICIP pp. 1733-1736 (2010). DOI 10.1109/ICIP.2010.5654434

16. Litjens, G., Kooi, T., Bejnordi, B.E., Setio, A.A.A., Ciompi, F., Ghafoorian, M., van der Laak, J.A., van Ginneken, B., Sánchez, C.I.: A survey on deep learning in medical image analysis. Medical Image Analysis 42(1995), 60-88 (2017). DOI 10.1016/j.media.2017.07.005

17. Moghbel, M., Mashohor, S., Mahmud, R., Saripan, M.I.B.: Review of liver segmentation and computer assisted detection/diagnosis methods in computed tomography. Artificial Intelligence Review 50(4), 497-537 (2018). DOI 10.1007/s10462-017-9550-x

18. Novikov, A.A., Lenis, D., Major, D., Hladuvka, J., Wimmer, M., Buhler, K.: Fully Convolutional Architectures for Multi-Class Segmentation in Chest Radiographs. IEEE Transactions on Medical Imaging pp. 1-13 (2018). DOI 10.1109/TMI.2018.2806086

19. O'Connor, J.P.B., Rose, C.J., Waterton, J.C., Carano, R.A.D., Parker, G.J.M., Jackson, A.: Imaging intratumor heterogeneity: Role in therapy response, resistance, and clinical outcome. Clinical Cancer Research 21(2), 249257 (2015). DOI 10.1158/1078-0432.CCR-14-0990

20. Oldhafer, K.J., Chavan, A., Frühauf, N.R., Flemming, P., Schlitt, H.J., Kubicka, S., Nashan, B., Weimann, A., Raab, R., Manns, M.P., Galanski, M.: Arterial chemoembolization before liver transplantation in patients with hepatocellular carcinoma: Marked tumor necrosis, but no survival benefit? Journal of Hepatology 29(6), 953-959 (1998). DOI 10.1016/S0168-8278(98)80123-2

21. Ronneberger, O., Fischer, P., Brox, T.: U-Net: Convolutional Networks for Biomedical Image Segmentation pp. 1-8 (2015). URL http://arxiv.org/abs/1505.04597

22. Ruskó, L., Bekes, G., Fidrich, M.: Automatic segmentation of the liver from multi- and single-phase contrastenhanced CT images. Medical Image Analysis 13(6), 871-882 (2009). DOI 10.1016/j.media.2009.07.009. URL http://dx.doi.org/10.1016/j.media.2009.07.009

23. Sadigh, G., Applegate, K.E., Baumgarten, D.A.: Comparative Accuracy of Intravenous Contrast-enhanced CT versus Noncontrast CT Plus Intravenous Contrast-enhanced CT in the Detection and Characterization of Patients with Hypervascular Liver Metastases. A Critically Appraised Topic. Academic Radiology 21(1), 113-125 (2014). DOI 10.1016/j.acra.2013.08.023. URL http://dx.doi.org/10.1016/j.acra.2013.08.023

24. Saito, A., Yamamoto, S., Nawano, S., Shimizu, A.: Automated liver segmentation from a postmortem CT scan based on a statistical shape model. International Journal of Computer Assisted Radiology and Surgery 12(2), 205-221 (2017). DOI 10.1007/s11548-016-1481-5

25. Segal, E., Sirlin, C.B., Ooi, C., Adler, A.S., Gollub, J., Chen, X., Chan, B.K., Matcuk, G.R., Barry, C.T. Chang, H.Y., Kuo, M.D.: Decoding global gene expression programs in liver cancer by noninvasive imaging. Nature Biotechnology 25(6), 675-680 (2007). DOI 10.1038/nbt1306

26. Shi, C., Cheng, Y., Liu, F., Wang, Y., Bai, J., Tamura, S.: A hierarchical local region-based sparse shape composition for liver segmentation in CT scans. Pattern Recognition 50, 88-106 (2016). DOI 10.1016/j.patcog. 2015.09.001. URL http://dx.doi.org/10.1016/j.patcog.2015.09.001

27. Sun, C., Guo, S., Zhang, H., Li, J., Chen, M., Ma, S., Jin, L., Liu, X., Li, X., Qian, X.: Automatic segmentation of liver tumors from multiphase contrast-enhanced CT images based on FCNs. Artificial Intelligence in Medicine 83, 58-66 (2017). DOI 10.1016/j.artmed.2017.03.008. URL http://dx.doi.org/10.1016/j.artmed.2017.03.008

28. Suzuki, K.: Overview of deep learning in medical imaging. Radiological Physics and Technology 10(3), 257-273 (2017). DOI 10.1007/s12194-017-0406-5

29. Warfield, S.K., Zou, K.H., Wells, W.M.: Simultaneous truth and performance level estimation (STAPLE): An algorithm for the validation of image segmentation. IEEE Transactions on Medical Imaging 23(7), 903-921 (2004). DOI 10.1109/TMI.2004.828354

30. Zhang, F., Yang, J., Nezami, N., Laage-gaupp, F., Chapiro, J., De Lin, M., Duncan, J.: Liver Tissue Classification Using an Auto-context-based Deep Neural Network with a Multi-phase Training Framework. In: W. Bai, G. Sanroma, G. Wu, B.C. Munsell, Y. Zhan, P. Coupé (eds.) Patch-Based Techniques in Medical Imaging, pp. 59-66. Springer International Publishing, Cham (2018)

31. Zheng, W., Thorne, N., Mckew, J.C.: Deep Learning in Medical Image Analysis. Annu Rev Biomed Eng 18, 1067-1073 (2015). DOI 10.1016/j.drudis.2013.07.001.Phenotypic 